\title{
EFFECTIVENESS OF A NEW TOOTHBRUSH DESIGN VERSUS A CONVENTIONAL TONGUE SCRAPER IN IMPROVING BREATH ODOR AND REDUCING TONGUE MICROBIOTA
}

\author{
Luciana Assirati CASEMIRO ${ }^{1}$, Carlos Henrique Gomes MARTINS ${ }^{2}$, Tatiane Cruz de CARVALHO ${ }^{3}$, Heitor PANZERI ${ }^{4}$, \\ Marco Aurélio Sichirolli LAVRADOR ${ }^{5}$, Fernanda de Carvalho Panzeri PIRES-DE-SOUZA ${ }^{6}$
}

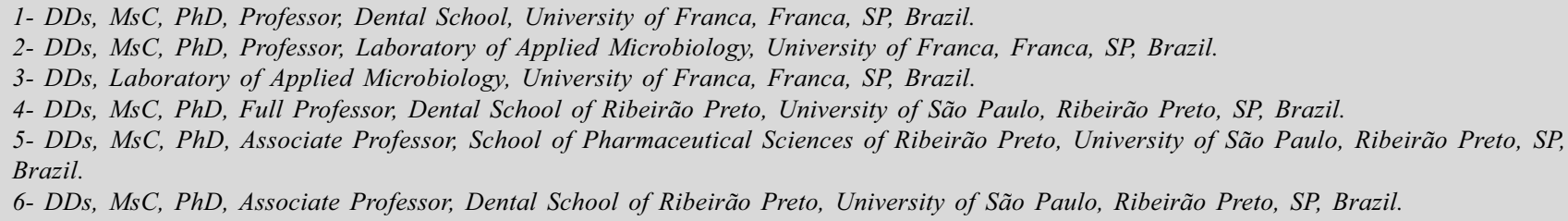

\begin{abstract}
$\boldsymbol{H}$ ( present study compared the effectiveness of a manual toothbrush with this new design, i.e., possessing a tongue scraper, and a commercial tongue scraper in improving breath odor and reducing the aerobic and anaerobic microbiota of tongue surface. The evaluations occurred at 4 moments, when the participants $(n=30)$ had their halitosis quantified with a halimeter and scored according to a 4-point scoring system corresponding to different levels of intensity. Saliva was collected for counts of aerobic and anaerobic microorganisms. Data were analyzed statistically by Friedman's test $(p<0.05)$. When differences were detected, the Wilcoxon test adjusted for Bonferroni correction was used for multiple comparisons (group to group). The results confirmed the importance of mechanical cleaning of the tongue, since this procedure provided an improvement in halitosis and reduction of aerobe and anaerobe counts. Regarding the evaluated methods, the toothbrush's tongue scraper and conventional tongue scraper had a similar performance in terms of breath improvement and reduction of tongue microbiota, and may be indicated as effective methods for tongue cleaning.
\end{abstract}

Key Words: Tongue biofilm. Tongue scraper. Toothbrush. Salivary bacterial count. Halimeter.

\section{INTRODUCTION}

The dorsum of the tongue is a large surface for oral accumulation of microorganisms and debris ${ }^{2,9,17}$. Biofilm is formed on tongue surface, being a dynamic structure composed by bacteria, epithelial cells scaled from oral mucosa, leukocytes from periodontal pockets, blood metabolites and different nutrients $\mathrm{s}^{3,7,26}$.

The existence of an association between tongue microorganisms and those present in saliva has been reported $^{13,15}$. The anaerobic microbiota of the tongue biofilm is one of the main responsible for the release of sulfur compounds, which are directly involved in the occurrence of halitosis. The microbial species isolated from the tongue include Porphyromonas gingivalis ${ }^{14}$, Aggregatibacter actinomycetemcomitans ${ }^{1,21}$, Spirochaetes ${ }^{14}$, Prevotella intermedia $^{4,5}$ and Capnocytophaga. ${ }^{12}$

Depending on the bacterial strain, the production of volatile sulfur compounds ${ }^{2,6,25}$, such as methyl mercaptan, hydrogen sulfide and dimethyl sulfide ${ }^{10,11,22,23}$, is associated with the tongue biofilm. These compounds are carried by exhaled air, contributing for the occurrence of halitosis ${ }^{6}$. The etiology of halitosis (from latin: halitus, that means exhaled air; and osis, that means pathological alteration) is varied. However, in $85 \%$ of the cases, periodontal pockets are the main responsible for this condition ${ }^{8}$. Some clinical conditions, such as fissured tongue, periodontal illness ${ }^{24}$, gastric reflux, prolonged fast and predominantly liquid or 
semi-solid diet, affect the rate of tongue biofilm formation and predispose the individual to halitosis ${ }^{16}$. Psychological factors also contribute to aggravate this scenario since salivary flow is reduced in this condition ${ }^{20}$.

In order to remove tongue biofilm and improve breath odor, specific instruments (tongue scrapers) or toothbrushes are used. New chemical and mechanical methods have also gained popularity for control of dental and tongue biofilm ${ }^{19}$. New toothbrush designs have also been developed with this purpose. This study compared the effectiveness of a new manual toothbrush that has a tongue scraper on the back of its head and a commercial tongue scraper in improving breath odor and reducing the aerobic and anaerobic microbiota of tongue surface.

\section{MATERIAL AND METHODS}

Thirty individuals ( 15 male and 15 female) aged 18 to 50 years with no evident pathological processes were enrolled in this crossover study. Eligible subjects could not have worn dentures or used medicines that cause xerostomy, mouthwashes or systemic antimicrobials for at least 1 month prior the study. Patients with conditions that cause breath alterations, such as diabetes mellitus, chronic renal illness, gastrointestinal disorders, cirrhosis, respiratory dysfunction, carcinomas and smoking, were excluded from the study ${ }^{7,16,18,23,24}$. All participants signed an informed consent form approved by the Research Ethics Committee of the University of Franca, Brazil (Protocol \#211/05).

Chemical and mechanical oral hygiene methods were prohibited $24 \mathrm{~h}$ before each evaluation session. Ingestion of alcoholic beverages or flavored foods was suspended. Approximately $1 \mathrm{~h} 30 \mathrm{~min}$ before the examination, the participants ate a light meal offered by the researchers. The volunteers were assigned to four groups, as follows: Group 1: The teeth and tongue were not cleaned; Group 2: The volunteers brushed their teeth with a toothbrush that has a tongue scraper on the back of its head (Condor Evolution, Condor, São Bento do Sul, SC, Brazil), but no tongue scraping was performed; Group 3: Toothbrushing was done (Condor Evolution) and the toothbrush's tongue scraper was used for $15 \mathrm{~s}$ on the dorsum of the tongue; Group 4: Toothbrushing was performed (Condor Evolution) and a commercial tongue scraper (Kolbe, Salvador, BA, Brazil) was used for $15 \mathrm{~s}$ on the dorsum of the tongue. The evaluations were performed at 1-week intervals. Toothbrushing was performed as usual. No flossing was performed.

Using a halimeter (BreathAlert, Tanita Corporation of America, Arlington Heights, IL, USA), halitosis was quantified according to a 4-point scoring system (1 - no halitosis, 2 - mild halitosis, 3 - moderate halitosis and 4 strong halitosis). After calibration following the manufacturer's instructions, the halimeter was given to the volunteer, who secured the device in the front of the mouth at a $1.0-\mathrm{cm}$ distance and exhaled air until the intensity level was recorded. This procedure was repeated three times for each participant.
Thereafter, two milliliter of non-stimulated saliva of each participant were collected and stored in sterile bottles with glass pearls. The maximum time elapsed between collection and laboratorial processing of the saliva samples was 10 min. The bottles with saliva were agitated in an automatic tubes agitator (Phoenix, São Paulo, SP Brazil) for 1 min and placed in an anaerobic chamber (MiniMac, Don Whitley Scientific, Bradford, UK). After, serial dilutions of $10^{-1}$ to $10^{-}$ ${ }^{4}$ in pre-reduced Schaedler broth (BBL, Nevada, CA, USA) were performed. For anaerobes, $50 \mathrm{~mL}$ of each dilution were seeded on the surface of Schaedler agar (BBL) supplemented with $5 \%$ of defibrinated sheep blood, $1 \mathrm{~mL} / \mathrm{L}$ of hemine (Sigma-Aldrich Corp., St. Louis, MO, USA) and $1 \mathrm{~mL} / \mathrm{L}$ of menadione (Sigma-Aldrich Corp.). For facultative aerobes, the same amounts of the dilutions were seeded on the surface of Schaedler agar (BBL) supplemented with 5\% of defibrinated sheep blood. For the growth of anaerobes, the plates were incubated in atmosphere of $10 \% \mathrm{H}_{2}, 10 \% \mathrm{CO}_{2}$ and $80 \% \mathrm{~N}_{2}\left(36^{\circ} \mathrm{C}, 3\right.$ days $)$ and for the growth of facultative and aerobic microorganisms, in aerobic atmosphere $\left(36^{\circ} \mathrm{C}, 2\right.$ days). After incubation, each plate was examined in a stereoscopic microscope (Nikon, Tokyo Japan) and the total number of colony forming units per milliliter of saliva (cfu/ $\mathrm{mL}$ ) was counted.

In both assays (quantification of halitosis and microbiological analysis), Friedman's test was used to determine the differences between the measures supplied by the halimeter and the bacterial counts of the groups. When statistically significant differences were detected among the groups, Wilcoxon's test adjusted with Bonferroni correction was used for multiple comparisons (group to group). Significance level was set at $5 \%$.

\section{RESULTS}

Comparing the values recorded with the halimeter, Group 1 (no toothbrushing or tongue cleaning) and Group 2 (toothbrushing alone) differed significantly from each other $(p<0.05)$. These groups were also significantly different from Groups 3 (toothbrush with a tongue scraper on the back of the head) and 4 (conventional tongue scraper) $(p<0.05)$. The adoption of tongue cleaning methods was associated with a decrease in the scores of halitosis recorded with the halimeter in a similar manner, as no statistically significant differences ( $p>0.05)$ were observed between Groups 3 and 4 (Table 1). There were no differences $(\mathrm{p}>0.05)$ between men and women regarding halitosis.

The results of this study indicate that toothbrushing per se did not alter significantly the breath odor. No statistically significant differences $(p>0.05)$ were found between the toothbrush's tongue scraper and the commercial tongue scraper, regarding their effectiveness in improving breath odor.

Regarding bacterial counts, Group 1 was statistically different from Group $2(\mathrm{p}<0.05)$, indicating that toothbrushing promoted a significant decrease in the number of microorganisms. Both groups differed significantly from 
TABLE 1- Halitosis scores recorded in each group

\begin{tabular}{lllll}
\hline & Group 1 & Group 2 & Group 3 & Group 4 \\
\hline Mean (standard deviation) & $4.0(0.00)$ & $3.6(0.49)$ & $2.8(0.40)$ & $2.7(0.59)$ \\
Median & 4 & 4 & 3 & 3 \\
Lowest value & 4 & 3 & 2 & 2 \\
Highest value & 4 & 4 & 3 & 4 \\
\hline
\end{tabular}

TABLE 2- Aerobic and anaerobic microbial counts (cfu/mL of saliva, log base 10)

Anaerobic microorganisms

Group 1 Group 2 Group 3 Group 4
Aerobic microorganisms

Group 1 Group 2 Group 3 Group 4

\begin{tabular}{lllllllll}
\hline Mean & 8.10 & 7.84 & 7.58 & 7.24 & 7.96 & 7.78 & 7.34 & 7.20 \\
(standard deviation) & $(0.53)$ & $(0.58)$ & $(0.92)$ & $(0.62)$ & $(0.46)$ & $(0.59)$ & $(0.91)$ & $(0.64)$ \\
Median & 7.81 & 7.64 & 7.39 & 6.85 & 7.76 & 7.65 & 7.11 & 6.90 \\
Lowest value & 7.55 & 7.39 & 6.41 & 6.54 & 7.39 & 6.84 & 6.38 & 6.54 \\
Highest value & 9.59 & 9.72 & 9.76 & 8.67 & 8.83 & 9.65 & 9.55 & 8.63 \\
\hline
\end{tabular}

Groups 3 and $4(\mathrm{p}<0.05)$, in which tongue cleaning methods were performed. However, Groups 3 and 4 presented statistically similar results to each other, demonstrating that both types of tongue scrapers had a similar performance in reducing the number of bacteria on tongue surface (Table $2)$. There were no differences $(p>0.05)$ between men and women regarding bacterial counts.

\section{DISCUSSION}

Several devices for daily tongue cleaning have been developed and used over time. Currently, it has been a trend to develop toothbrushes that have not only bristles for dental cleaning, but also projections on the back of their head that act as a tongue scraper. In the present study, a toothbrush with this new design and a conventional tongue scraper commercialized in Brazil were compared. The results showed that toothbrushing alone (Group 2) did not improve significantly the breath odor of the participants compared to no tooth/tongue cleaning (Group 1), as demonstrated by the high incidence of scores 4 in Group 2 (mean score = 3.6) (Table 1). Therefore, tongue cleaning is important to improve breath odor ${ }^{2,6,25}$ mainly considering that oral conditions are determinant for halitosis in about $85 \%$ of the cases ${ }^{8}$.

The tongue cleaning methods evaluate in the present study (toothbrush's tongue scraper - Group 3 and commercial tongue scraper - Group 4) had a similar effect on the participants' halitosis (Table 1). These groups presented mean scores of 2.8 and 2.7 , respectively. It is likely that due to the halimeter accuracy, the recorded scores were higher, not being $<2$ in any situation. In spite of these values, individuals with characteristics that contribute to increase halitosis $7,16,18,23,24$ were not included in this study and hence the use of a device to remove tongue biofilm was proved effective in improving breath odor.

Regarding to bacterial counts, no significant differences ( $p>0.05$ ) were found between groups 3 and 4 . All other group pairs differed significantly from each other. These results demonstrate that both methods of tongue cleaning were equally satisfactory for reduction of the total number of microorganisms from tongue surface. It was also observed that there was a reduction of the total number of cfu when toothbrushing and tongue cleaning were performed. Counting of salivary bacteria for evaluation of mechanical methods of tongue cleaning is justified since the removal of bacterial niches, as those present on tongue surface, contributes to reduce the total number of bacteria of oral cavity $^{7}$. Moreover, the incubation of the collected saliva in aerobiosis and anaerobiosis is justified because strict anaerobic, aerobic and facultative aerobic bacteria present on the dorsum of the tongue are usually isolated in saliva ${ }^{13,15}$.

In the present study, data from both assays indicate that tongue scraping is an essential procedure to reduce tongue microbiota and release of volatile sulfur compounds. Both devices (toothbrush's tongue scraper and commercial tongue scraper) were similarly effective. The results of each group may have been influenced by variations in biofilm formation rate, which is affected by factors, such as diet (quality and amount) during the course of the study. Thus, is not possible to assure that the amount and quality of bacteria present on the dorsum of the tongue was the same at all evaluation periods, which may be considered a limitation of this study. In spite of this, the results reinforce the importance of using mechanical methods for tongue cleaning. It was also demonstrated that the reduction of the 
number of aerobes and anaerobes from tongue surface improved breath odor. It was confirmed that the exhaled volatile sulfur compounds, that contribute to halitosis ${ }^{6}$, are related to tongue microbiota, as reported elsewhere ${ }^{2,6,10,11,22,23,25}$. Both cleaning methods evaluated in this study may be indicated as instruments for removal of tongue biofilm.

\section{CONCLUSIONS}

The findings of the present study demonstrate that the adoption of methods for tongue cleaning associated to toothbrushing minimizes halitosis and reduces bacterial counts on tongue surface. The evaluated methods (toothbrush with a tongue scraper on the back of its head and conventional tongue scraper) were equally effective in the improving breath odor and reducing the facultative aerobic and anaerobic microbiota on tongue surface of the studied population.

\section{REFERENCES}

1- Asikainen S, Lai CH, Alaluusua S, Slots J. Distribution of Actinobacillus. actinomycetemcomitans serotypes in periodontal health and disease. Oral Microbiol Immunol. 1991;6(2):115-8.

2- Bosy A, Kulkarni GV, Rosenberg M, McCulloch CAG. Relationship of oral malodor to periodontitis: evidence of independence in discrete subpopulations. J Periodontol. 1994;65(1):37-46.

3- Chen ZL. Brief history of tongue inspection. Chin Med J. 1987;100(1):38-44.

4- Danser MM, van Winkelhoff J, de Graaff J, van der Velden U. Putative periodontal pathogens colonizing oral mucous membranes in denture-wearing subjects with a past history of periodontitis. J Clin Periodontol. 1995;22(11):854-9.

5- Danser MM, van Winkelhoff J, de Graaff J, Loos BG, van der Velden U. Short-therm effect of full-mouth extraction on periodontal pathogens colonizing the oral mucous membranes. J Clin Microbiol. $1994 ; 21(7): 484-90$

6- De Boever EH, Loesche WJ. Assessing the contribution of anaerobic microflora of the tongue to oral malodor. J Am Dent Assoc. $1995 ; 126(10): 1384-93$

7- Mantilla Gómez S, Danser MM, Sipos PM, Rowshani B, van der Velden U, van der Wijden GA. Tongue coating and salivary bacterial counts in healthy/gingivitis subjects and periodontitis patients. J Clin Periodontol. 2001;28(10):970-8.

8- Hine HK. Halitosis. J Am Dent Assoc. 1957;55(1):37-46

9- Jacobson SE, Crawford JJ, McFall WR. Oral physiotherapy of the tongue and palate: relationship to plaque control. J Am Dent Assoc. 1973;87(1):134-9.

10 - Jenkins GN. Sensations arising in the mouth. In: Jenkins GN Physiology and biochemistry of the mouth. London: Blackwell Scientific Publications; 1978. p. 542-70.

11 - Kleinberg I, Westbay G. Oral malodor. Crit Rev Oral Biol Med. $1990 ; 1(4): 247-59$.
12- Könönen E, Asikainen S, Alaluusua S, Könönen M, Summanen P, Kanervo A, et al. Are certain oral pathogens part of normal oral flora in denture-wearing edentulous subjects? Oral Microbiol Immunol. $1991 ; 6(2): 119-22$.

13-Krasse B. The proportional distribution of Streptococcus salivarius and other streptococci in various parts of the mouth. Odontol Revy. 1954;5(3):203-11

14- Lee KH, Tanner ACR, Maiden MFJ, Webwer HP. Pre-and postimplantation microbiota of the tongue, teeth, and newly-placed implants. J Clin Periodontol. 1999;26(12):822-32.

15- Lindquist B, Emilson CG, Wennerholm K. Relationship between mutans streptococci in saliva and their colonization of the tooth surfaces. Oral Microbiol Immunol. 1989;4(2):71-6.

16- Massler M, Emslie RD, Bolden TE. Fetor ex ore: a review. Oral Surg Oral Med Oral Pathol. 1951;4(1):110-25.

17- Massler M. Geriatric dentistry: root caries in the elderly. J Prosthet Dent. $1980 ; 44(2): 147-9$.

18-Morita M, Musinski DL, Wang HL. Assessment of newly developed tongue sulfide probe for detecting oral malodor. J Clin Periodontol. 2001;28(5):494-6.

19- Myatt GJ, Hunt SA, Barlow AP, Winston JL, Bordas A, El Maaytah M. A clinical study to asses the breath protection efficacy of denture adhesive. J Contemp Dent Pract. 2002;3(4):1-10.

20-Oho T, Yoshida Y, Shimazaki Y, Yamashita Y, Koga T. Psychological condition of patients complaining of halitosis. J Dent. 2001;29(1):31-3.

21 - Timmerman MF, van der Weijden GA, Armand S, Abbas F, Winkel $\mathrm{EG}$, van Winkelhoff AJ, et al. Untreated periodontitis disease in Indonesian adolescents: clinical and microbiological baseline data. J Clin Periodontol. 1998;25(3):215-24.

22 - Tonzetich J. Direct gas chromatographic analysis of sulphur compounds in mouth air in man. Arch Oral Biol. 1971;16(6):58797

23- Tonzetich J. Production and origin of malodor: a review of mechanisms and methods of analysis. J Periodontol. 1977;48(1):1320 .

24- Yaegaki K, Sanada K. Volatile sulfur compounds in mouth air from clinically healthy subjects and patients with periodontal disease. J Periodontal Res. 1992;27(4 Pt 1):233-8.

25 - Yaegaki K, Sanada K. Effects of a two-phase oil-water mouthwash on halitosis. Clin Prev Dent. 1992;14(1):5-9. 